\title{
Characterization of Multi-agent systems: A systematic mapping study
}

\section{Caracterización de Sistemas multi-agente: Un estudio de mapeo sistemático}

\author{
Diego Fernando Andaluz Espinosa, Patricio Alejandro Molina Palma, and José \\ Rubén Caiza Caizabuano \\ Universidad de las Fuerzas Armadas, Cotopaxi, Ecuador
}

\section{Abstract}

Multi-agent systems have gained a lot of interest in articles, conferences and magazines. However, it is still a recent issue which is not understood or the scope of it is unknown. The objective of this work is to investigate in more detail the articles and to build a preliminary classification system to structure the field of Multi-agent systems. As a

Corresponding Author: Diego Fernando Andaluz Espinosa

dfandaluz@espe.edu.ec

Received: 24 December 2019

Accepted: 2 January 2020

Published: 8 January 2020

Publishing services provided by Knowledge E

(c) Diego Fernando Andaluz Espinosa et al. This article is distributed under the terms of the Creative Commons

Attribution License, which permits unrestricted use and redistribution provided that the original author and source are credited.

Selection and Peer-review under the responsibility of the SIIPRIN-CITEGC Conference Committee. result, an overview of this area of research can be obtained through the response to the research questions posed.

Resumen. Los sistemas multi-agente han ganado un interés muy grande en artículos, conferencias y revistas. Sin embargo sigue siendo un tema reciente el cual no se comprende ni se conoce los alcances del mismo. Este trabajo tiene como objetivo investigar con más detalle los artículos y construir un sistema de clasificación preliminar para estructurar el campo de los sistemas Multi-agente. Como resultado, se espera proporcionar una visión general de esta área de investigación a través de responder a las preguntas de investigación planteadas. Creemos que el presente trabajo será una herramienta que encontrará ámbitos todavía aun no investigados.

Keywords: Intelligent agent, multi-agent, intelligent system, artificial intelligence, distributed system, autonomy, agent-based systems, enterprise y/o industry.

\section{Introducción}

El incremento de la funcionalidad y la autonomía de los programas se han convertido en una necesidad imperante en los últimos años. Incorporar métodos intuitivos y predictivos en su diseño es el reto de las tecnologías modernas. El objetivo a lograr es la creación de entes o sistemas autónomos que sean capaces de llevar a cabo tareas y funciones que apliquen razonamiento y aprendizaje [1].

Wooldridge y Jennings definen a los sistemas multi-agente (MAS) como paradigma de ingeniería de software para el diseño y desarrollo de sistemas de software complejos 
[2]. En un sistema multi-agente, los agentes se comunican entre sí con el fin de alcanzar y mejorar sus propias metas o las metas del sistema donde éstos existen [3].

La comunicación entre los agentes se establece mediante un proceso de negociación que involucra a varios agentes con intereses comunes. Además, ofrece perspectivas sobre cómo mantener un equilibrio entre el comportamiento reactivo y proactivo en situaciones complejas y dinámicas [2]. Cada agente cumple una función dentro del conjunto de roles. La asignación basada en agentes implica el uso de estos agentes para realizar las actividades necesarias durante la negociación, así como para representar a los diferentes actores involucrados [4]. Es por esta razón que es importante determinar los estudios científicos que se han realizado en este ámbito.

A la fecha existen varios trabajos de investigación en los que se documenta el uso de sistemas multi-agente. En este contexto cada uno de estos trabajos contribuye con herramientas, modelos, metodologías y enfoques. Así por ejemplo Algarvio, Lopes, \& Santana aportan con un modelo para ayudar a gestionar la complejidad de los mercados energéticos [5]. Hsieh \& Lin proponen una metodología de solución para la programación de flujos de trabajo en sistemas multi-agente (MAS) [6]. Lopes, Algarvio, $\&$ Coelho proporcionan una herramienta orientada al uso de agentes de software en el comercio bilateral de la electricidad [7].

Parunak y Odell, se centra en intentar adaptar herramientas de desarrollo ya existentes y que están teniendo éxito para aplicaciones industriales reales, como es el caso de UML, tratando de orientarlas hacia el campo de los agentes [8].

Como un ejemplo de un sistema complejo y distribuido, considere la tarea de aprovisionar dinámicamente a un público red de comunicación (como Internet) como una red privada virtual para usuarios finales, concretamente una reunión de videoconferencia [9], Es en este entorno es que la aplicación de sistemas multi-agente ayuda a tareas de distribución y negociación para optimizar el proceso.

En particular en este artículo se aplica un estudio de mapeo, para recopilar y sintetizar evidencia científica de los sistemas multi-agentes, con el fin de proponernos preguntas y sub preguntas de investigación y dar respuesta a las mismas.

Un paso esencial de cualquier estudio es la definición de las preguntas de investigación, ya que estas permitirán la organización y ejecución del proceso en este caso el estudio de mapeo planteado [10]. Por lo tanto, con el fin de abordar los problemas descritos anteriormente, este estudio de mapeo se centrará en la respuesta a la siguiente pregunta: 
1) Cómo el campo de los sistemas multi-agente se encuentra estructurado y la madurez de las contribuciones. En función de esta pregunta se plantea las siguientes sub preguntas:

1.1) Que áreas de los sistemas multi-agente se han abordado y como los artículos analizados se distribuyen a través de esas áreas.

1.2) Qué tipos de trabajo son publicados en la zona y en particular que tipo de evaluación y novedad constituyen.

Las preguntas de investigación se establecieron considerando los elementos población, intervención y resultados como lo propone [11].

El presente estudio explora un tema amplio y clasifica los trabajos de investigación primarios en ese dominio específico [12]. Por lo que, los resultados de este artículo se espera que sean un aporte significativo para la comunidad científica al proporcionar una descripción general de la literatura en el tema planteado [13]

El presente artículo está estructurado de la siguiente manera: La Sección 2 describe la metodología del estudio. La Sección 3 sintetiza las contribuciones encontradas en la literatura en respuesta a nuestra primera pregunta de investigación. Se realiza un análisis de la información y se establece una panorámica para los investigadores. Finalmente, en la sección 4 se encuentra la discusión y las conclusiones.

\section{Método}

El estudio de mapeo de la literatura es un método de estudio secundario que ha recibido mucha atención últimamente y es inspirado en la investigación médica [14] y que se ha aplicado exitosamente en áreas como la ingeniería del software. Este estudio de mapeo sistemático (EMS) hace una revisión de los estudios primarios en un tema específico, con el fin de identificar la evidencia disponible en el tema [12]. El mapeo realizado buscó evidencia de las distintas perspectivas del tema y se basa en una metodología propuesta por Petersen y adoptada e interpretada por muchos autores e investigadores, indicando los siguientes pasos [15]:

1. Definición de las preguntas de investigación.

2. Búsqueda de la literatura relevante.

3. Selección de los estudios pertinentes

4. Clasificación de los artículos

5. Extracción y agregación de los datos. 
Autores como Petersen presentan una descripción detallada de cómo realizar estudios de mapeo. Por lo que este estudio tendrá como guía el esquema mostrado en la Fig. 1.

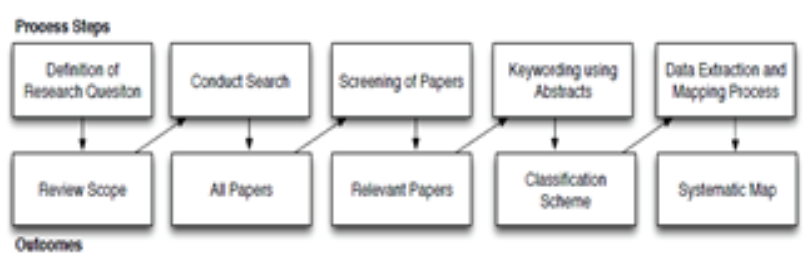

Figure 1: Proceso de estudio de mapeo. La imagen define el proceso de estudio de mapeo según Petersen: 1) Definición de preguntas de investigación. 2) Búsqueda de estudios primarios. 3) Selección de documentos para su inclusión y exclusión. 4) Palabras clave de los resúmenes. 5) Extracción de datos y mapeo de estudios. Origen: [16].

Un mapeo es considerado por muchos como un paso previo e imprescindible para decidir en qué áreas en concreto del campo es interesante abordar una revisión sistemática más detallada [17].

\section{Estudio de Mapeo}

\subsection{Definición de preguntas de investigación}

Esta investigación tiene como objetivos generales, dar respuestas inteligibles, confiables y válidas a preguntas específicas. Toda investigación comienza pues, con algún tipo de interrogante que tratará de ser resuelta [18]. Este estudio pretende responder a las siguientes preguntas de investigación:

Q1. Como el campo de los sistemas multi-agente se encuentra estructurado y la madurez de las contribuciones.

Q1.1 Qué áreas de los sistemas multi-agente se han abordado y cómo los artículos analizados se distribuyen a través de esas áreas.

Q1.2 Que tipos de trabajo son publicados en la zona y en particular que tipo de evaluación y novedad constituyen.

\subsection{Búsqueda de estudios primarios.}

Para realizar una revisión de la literatura que permita identificar estudios relevantes se sugiere cinco criterios conocidos como Población, Intervención, Comparación, Resultados y Contexto [19]. La estrategia PICOC apoya en estas definiciones pues, orienta para la construcción de las preguntas de investigación y la búsqueda de bibliografía. 
El presente estudio se ha estructurado en términos de población, intervención, comparación y resultado [14], como lo muestra la tabla 1.

TABLE 1: Determinación de la población, la intervención y los resultados.

Factores
Población
Intervención
Resultados

\begin{tabular}{c}
$\begin{array}{c}\text { Términos principales de la } \\
\text { Investigación }\end{array}$ \\
Industrias o Empresas \\
Sistemas multi-agentes \\
\hline Asignación y negociación de tareas
\end{tabular}

Sinónimos
Factories, manufactures.
Intelligent agent, multi agent,
intelligent system, autonomous
system.
Assignment, distribute, negotiate,
deal, trade, divide, Split.

Es importante evitar el sesgo y que el estudio quede incompleto. Para lo cual se debe considerar todos los documentos, ya que algunos subtemas podrían estar sobrerrepresentados o subrepresentados para ciertos métodos de estudio.

La elección de las bases de datos científicas es un punto importante a la hora de realizar las búsquedas. En este estudio, se utilizaron varias fuentes de datos según lo sugerido por Emam Hossain [20]. La tabla 2 muestra una lista de las fuentes de bases de datos electrónicas que se han utilizado para descubrir las respuestas a las preguntas de investigación.

TABLE 2: Bases de Datos Científicas.

Origen
Scopus
IEEE
Science Direct

URL
https://www.scopus.com/search
https://ieeexplore.ieee.org/
https://www.sciencedirect.com

\begin{tabular}{|c|} 
Nro. Publicaciones \\
$\qquad 92$ \\
21 \\
8 \\
\hline
\end{tabular}

Para el análisis de los sistemas multi-agente, se tuvieron en cuenta todos los resultados de las bases de datos relevantes para las ciencias de la computación. Sin embargo, sólo se consideró artículos de revistas como Engineering Applications of Artificial Intelligence, Advanced Engineering Informatics, IEEE Transactions on Automation Science and Engineering entre otras. Las cuales tienen un factor de impacto considerable en la investigación. El factor de impacto de las revistas es un indicador presente en casi todos los estudios bibliométricos [21].

\subsection{Palabras Clave}

En [22] se describe esquemas de clasificación de artículo, tipos de estudio entre otros, pero no menciona un esquema de clasificación. Para nuestro estudio, seguimos un 
prototipo propuesto por [23] en el que las palabras clave juegan un rol preponderante en la clasificación de artículos, Este razonamiento se refleja en los strings de búsqueda:

Title-abs-key ("intelligent agent" or "multi agent" or "intelligent system" or "autonomous system") and title-abs-key (task or work or job) and title-abs-key (techni* or method* or strateg* or model) and title-abs-key (autonomia or intelligencia and title-abs-key (industry or enterprise).

\subsection{Selección de documentos para su inclusión y exclusión.}

En la tabla 3 se muestra los criterios de inclusión y exclusión que se utilizó para excluir los estudios que no son relevantes a la hora de responder las preguntas de investigación..Nos pareció útil excluir los trabajos que sólo concuerdan con las palabras clave pero tienen un contexto lejos del tema estudiado. Esta técnica es un prototipo planteado en [23] y no encontró ninguna clasificación errónea a causa de ella. Adicionalmente se muestra los criterios de selección, los cuales nos permiten hacer énfasis en el análisis de esos documentos. Los criterios de inclusión, exclusión y selección permiten una adecuada selección de las publicaciones y que sean un aporte significativo al estudio [24] y [25].

Este es un buen punto de partida para determinar el esquema de clasificación y la distribución de los artículos entre las categorías identificadas.

En la tabla 4 se muestra el umbral de factor de impacto que se consideró como política de inclusión y exclusión.

\subsection{Esquema de clasificación.}

Varios autores describen que para un estudio de mapeo se requiere establecer un esquema de clasificación [16, 26]. Aquí, el uso de palabras clave es una manera de reducir el tiempo necesario para desarrollar el esquema de clasificación y asegurar que el esquema tenga en cuenta los estudios existentes [23], vea la Fig. 2.

Los revisores leen resúmenes y buscan palabras clave y conceptos que reflejen la contribución al documento. De la exploración inicial se busca conceptos que reflejen los aportes al artículo. Al hacerlo, el revisor también identificó el contexto de la investigación. Se mejoró la cadena de búsqueda con palabras clave similares y nuevas. Del conjunto de artículos se categorizó y organizó. Los estudios se seleccionan mediante el siguiente esquema:

Contexto: Indica en qué tipo de actividad se aplica los sistemas multi-agente. 
TABLE 3: Criterios de inclusión, exclusión y selección.

\begin{tabular}{|c|c|c|}
\hline Id & Tipo & Descripción \\
\hline $\mathrm{Cl1}$ & Inclusión & $\begin{array}{l}\text { Los términos más relevantes aparecen en el abstract y } \\
\text { en los keywords }\end{array}$ \\
\hline $\mathrm{Cl} 2$ & Inclusión & $\begin{array}{l}\text { El estudio primario menciona características } \\
\text { relacionadas a las palabras clave }\end{array}$ \\
\hline $\mathrm{Cl} 3$ & Inclusión & $\begin{array}{l}\text { Los estudios se encuentran en relación a las } \\
\text { preguntas de investigación. }\end{array}$ \\
\hline $\mathrm{Cl} 4$ & Inclusión & $\begin{array}{l}\text { Los tipos de documentos son artículos, conferencias, } \\
\text { conferencias review }\end{array}$ \\
\hline $\mathrm{Cl} 5$ & Inclusión & $\begin{array}{l}\text { El origen de los artículos provienen de journals, } \\
\text { conferencias y books }\end{array}$ \\
\hline $\mathrm{Cl6}$ & Inclusión & $\begin{array}{l}\text { Los artículos son producto de búsquedas en bases de } \\
\text { datos científicas Scopus, IEEE y ScienceDirect }\end{array}$ \\
\hline CE1 & Exclusión & $\begin{array}{l}\text { Se han descartado los artículos menores al } 2010 \text { por } \\
\text { considerarse desactualizados. }\end{array}$ \\
\hline CE2 & Exclusión & $\begin{array}{l}\text { El estudio primario está en un contexto diferente a lo } \\
\text { que se investiga }\end{array}$ \\
\hline CE3 & Exclusión & No se puede tener acceso al artículo completo. \\
\hline CS1 & Selección & Se aplican criterios de inclusión y de exclusión \\
\hline CS2 & Selección & $\begin{array}{l}\text { Se toma documentos que aportan con métodos, } \\
\text { técnicas, modelos de negociación y división de tareas }\end{array}$ \\
\hline cs3 & Selección & $\begin{array}{l}\text { Los artículos previamente obtenidos serán refinados } \\
\text { en base a una lectura diagonal del artículo }\end{array}$ \\
\hline CS4 & Selección & $\begin{array}{l}\text { Se pone como prioridad los artículos más relevantes, } \\
\text { es decir que tienen un mayor impacto. }\end{array}$ \\
\hline
\end{tabular}

TABLE 4: Umbral de factor de impacto de los artículos.

\begin{tabular}{|l|c|c|}
\hline Porcentaje & Cuartil & Promedio SJR \\
\hline $\mathbf{7 1 . 4 3 \%}$ & Q1 & 1.36 \\
\hline $\mathbf{0 4 . 7 6} \%$ & Q2 & 1.43 \\
\hline $\mathbf{1 4 . 2 9} \%$ & Q3 & 0.30 \\
\hline $\mathbf{0 9 . 5 2} \%$ & Q4 & 0.18 \\
\hline
\end{tabular}

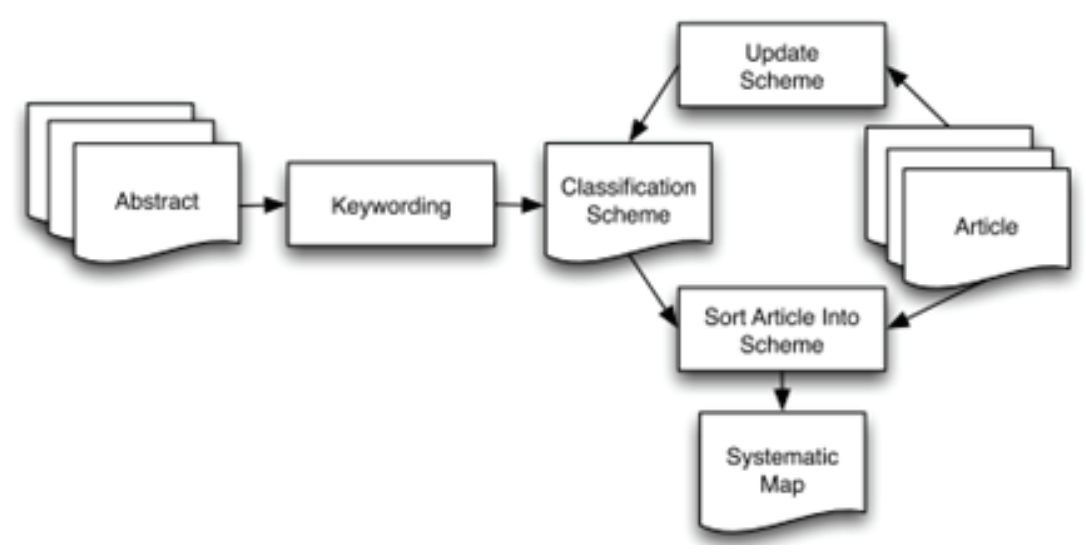

Figure 2: Esquema de clasificación. 
Tipo de contribución: Describe que se desarrolló para lograr avances en el área de enfoque. Las posibles contribuciones son procesos, métodos, modelos, métricas y herramientas [26].

Tipo de investigación: Es el nivel de novedad y cómo se caracteriza la investigación. Wieringa et al proponen la clasificación de los trabajos en investigación de validación, investigación de evaluación, propuesta de soluciones, trabajos filosóficos, trabajos de opinión y trabajos de experiencia [27].

Nivel de contribución: ALTA, MEDIA, BAJA (un estudio puede aportar información que responda a una o más preguntas de investigación).

\subsection{Filtro y Organización de artículos}

En primer lugar se identificó un conjunto inicial de artículos científicos a través de búsquedas exploratorias en bases de datos científicas, La cadena de búsqueda contiene palabras clave "multi-agente", "inteligencia artificial" y "autonomía" que se aplican sólo a los metadatos, lo que ha demostrado ser suficiente en estudios de mapeo [28]. De esta primera búsqueda obtuvimos ocho documentos tales como [29] los cuales fueron examinados para obtener una visión general del área; desafíos discutidos con frecuencia, clasificaciones de uso común y palabras clave importantes [30--32].

Luego se decidió ampliar la cadena de búsqueda con sinónimos como "Sistemas autónomos", “Agentes Inteligentes", “Asignación", “técnicas", "Modelos". Con esta nueva cadena de búsqueda se pudo incrementar el número de documentos científicos a doscientos doce artículos. Desde este punto de vista, la selección de características relevantes, y la eliminación de las irrelevantes, es uno de los problemas centrales en nuestro estudio [33]. Sólo se incluyeron artículos con un enfoque claro y publicado desde el año 2010.

Posteriormente se procede a definir los estudios primarios de nuestra investigación, en base a una lectura más profunda en la introducción de los documentos. Luego de este paso el número de documentos se ha reducido a noventa y dos. Las publicaciones excluidas fueron aquellas que no cumplieron criterios de selección como lo analizaremos más adelante.

Finalmente se realizan búsquedas en Scopus, IEEE, y ScienceDirect, dándonos la seguridad de ser documentos revisados por pares especialistas en el área de conocimiento. Por lo tanto, creemos que la búsqueda de publicaciones es suficientemente amplia y ofrece una buena imagen del estado del arte en la investigación sistemas multi-agente. 
Mediante la aplicación de los criterios de selección se ha logrado tener un conjunto de cuarenta y dos artículos que ayudan a construir nuestro estudio de mapeo y dar respuesta a las interrogantes de investigación. Los criterios de inclusión/exclusión se relacionan directamente con los títulos de los artículos [16].

El presente estudio ha identificado ochenta y nueve estudios primarios que tienen contribución a los objetivos de investigación. En la Fig. 3 se muestra la distribución de estos estudios según las bases científicas de información.

\section{BASES CIENTIFICAS}

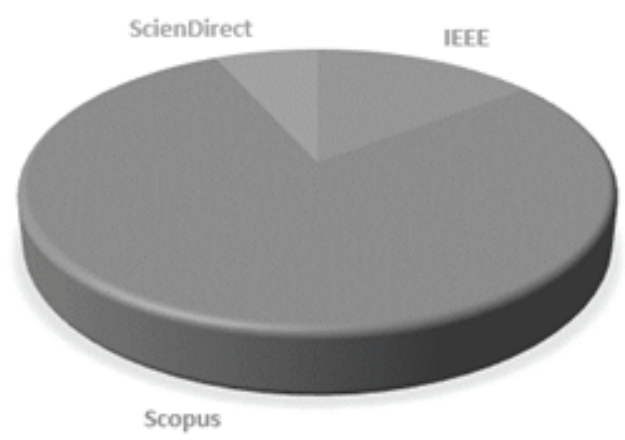

Figure 3: Distribución de las publicaciones en las bases de datos científicas.

Se ha creído conveniente identificar cuantos aportes significativos o de nivel alto se establecen en el presente estudio, esto nos ayuda a tener una óptica de la calidad de los artículos, para lo cual se puede observar en la Fig. 4.

\section{NIVEL DE CONTRIBUCIONES}

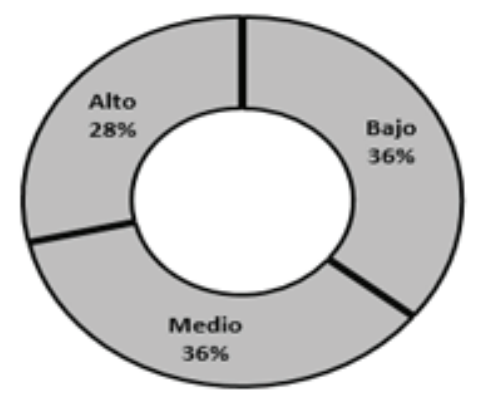

Figure 4: Niveles de contribución: Alto 28\%, Medio 36\% y Bajo 36\%.

Podemos observar que existe un buen número de aportes (28\%) considerados significativos, ya que tienen una contribución alta en cuanto a los objetivos de esta investigación. 


\section{Análisis de Resultados}

En la búsqueda de una respuesta a la interrogante Q1: ¿Como el campo de los sistemas multi-agente se encuentra estructurado y la madurez de las contribuciones?, se ha procedido al análisis de los 42 documentos que pasaron los filtros de selección, encontrando los tipos de contribución mostrados en la tabla 5.

TABLE 5: Tipos de Contribución.

Contribución
Proceso
Metodología
Modelo
Herramienta
Descripción del área

\begin{tabular}{|c|}
\hline Número \\
\hline 9 \\
\hline 8 \\
\hline 9 \\
10 \\
\hline 6 \\
\hline
\end{tabular}

\begin{tabular}{|c|}
\hline$\%$ \\
\hline 21.43 \\
\hline 19.05 \\
\hline 21.43 \\
\hline 23.81 \\
\hline 14.29 \\
\hline
\end{tabular}

Para esta clasificación se ha tomado como referencia los parámetros propuestos en [13] los cuales clasifican a los tipos de estudio como Proceso, Metodología, Modelo, Herramienta y descripción del Área. Las contribuciones que son de tipo proceso aporta un $21.43 \%$, los tipos metodología aporta con $19.05 \%$, las de tipo modelo es $21.43 \%$, los tipos herramienta es de $23.81 \%$ y las contribuciones que son de tipo descripción de área es de un $14.5 \%$.

Continuamos con el proceso de categorización de los estudios pertinentes para lo que se procede a establecer una clasificación en función de parámetros. [13, 26, 34] proponen esquemas de clasificación por tipo de investigación. Para el presente trabajo se considerará los tipos de investigación siguientes: Experimento, Caso de estudio, Observación, Encuesta, informe de experiencia e investigación. En la tabla 6 se muestra un resumen de las publicaciones científicas en función de los tipos de investigación que se han realizado para este estudio.

TABLE 6: Tipos de Investigación.

\begin{tabular}{|l|c|c|}
\hline Tipo & Frecuencia & Porcentaje \% \\
\hline Experimento & 9 & 21.43 \\
\hline Caso de Estudio & 15 & 35.71 \\
\hline Observación & 2 & 4.76 \\
\hline Encuesta & 1 & 2.38 \\
\hline Experiencia & 7 & 16.67 \\
\hline Investigación & 8 & 19.05 \\
\hline Total & 42 & 100.00 \\
\hline
\end{tabular}


Con los datos obtenidos en las tablas 4 y 5 cruzamos información con la finalidad de identificar los tipos de investigación que existe por cada tipo de contribución.

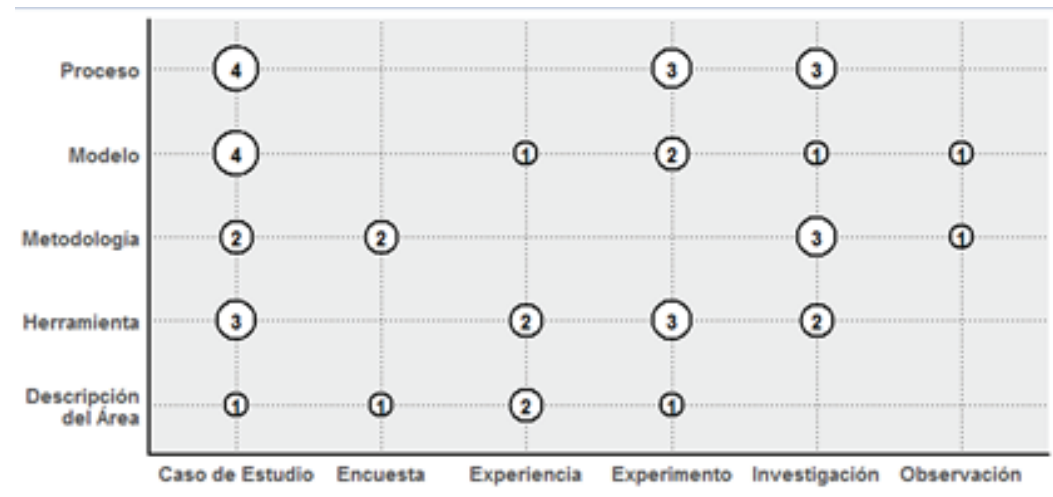

Figure 5: Tipos de contribuciones.

\subsection{En lo que respecta a las contribuciones de tipo Procesos}

[35] menciona que ha desarrollado un sistema multi-agente (MASCEM), que simula el entorno de los mercados de electricidad. En [36] indica que el problema de coordinación son los problemas clave de la gestión de la cadena de suministro, con el fin de promover la coordinación entre las cadenas de suministro, en este documento se acumula las autonómicas de negociación modelos en ontología, en la negociación y la oferta, en el software basado en $\mathrm{M}$ - agente.

En [37] se está investigando el uso de un enfoque que puede beneficiarse de las ventajas relativas de las soluciones de PLC y agentes. Ya que se presenta una nueva arquitectura híbrida que combina las funcionalidades de un PLC con las de los agentes industriales. En [38] muestra cómo estos mercados aún no implementados, altamente automatizados e interconectados aliviarán los problemas comúnmente asociados con los intermediarios existentes y proporcionarán apoyo a los agentes de software autónomos y descentralizados para realizar subastas contractualmente vinculantes de servicios de transporte multimodal de carga utilizando una subasta combinatoria bilateral. Mientras que [5] menciona que el objetivo de este trabajo es utilizar agentes de software autónomos para ayudar a gestionar la complejidad de los mercados minoristas, explicando que la industria de la electricidad ha sufrido cambios enormes y las fuerzas del mercado impulsan ahora el precio de la energía, este documento se centra en los mercados minoristas en los que los clientes de uso final pueden elegir su proveedor de electricidad como caso de estudio. [39] es un experimento de aviación donde, la dinámica de la red cooperativa de seguimiento resultante se describe mediante un nuevo modelo de sistemas impulsivos. Dando 
como resultado, para garantizar la estabilidad del sistema de red de circuito cerrado, las ganancias de los controladores se eligen de forma efectiva utilizando la solución de una ecuación algebraica de Riccati, en el cual proporcionan una estrategia de control distribuido para cada agente móvil en una red de vigilancia en el avión para perseguir de manera cooperativa a un evasor. La tarea de seguimiento se transmite de un agente a otro cuando el evasor cruza el límite de las regiones de Voronoi divididas de acuerdo con las posiciones de los agentes.

El trabajo [40] es un experimento enfocado en la robótica que menciona la creación de trayectorias libres de colisiones para robots móviles, conocido como el problema de planificación de ruta, se considera uno de los problemas básicos de la robótica, en el caso de sistemas robóticos múltiples, la complejidad de dichos sistemas aumenta proporcionalmente con el número de robots, debido al hecho de que todos los robots deben actuar como una unidad para completar una tarea compuesta, en un equipo de robot cooperativo. Tratando la combinación de autómatas celulares y colonias artificiales de hormigas para trayectos libres de colisiones.

El artículo [41] es un caso de estudio en el campo de la robótica en el cual manifiesta que para implementar el seguimiento automático de objetivos, evitar obstáculos y evitar colisiones entre los miembros del grupo, el marco de control se divide en tres capas en función de las asignaciones de tareas: diseño de la estrategia, planificación del movimiento y diseño de entrada de control.

Es importante mencionar que todos estos trabajos de investigación se orientan a automatizar procesos complejos en donde los agentes cooperan para cumplir un objetivo en común.

\subsection{En lo que respecta a contribuciones de tipo Metodología}

El estudio [5] tiene como objetivo utilizar agentes de software autónomos para ayudar a gestionar la complejidad de los mercados minoristas en la industria eléctrica. En [42] se realiza un experimento en el que se presenta una arquitectura auto organizada asistida por la nube (CASOA) que comprende agentes inteligentes y la nube, para comunicarse y negociar a través de redes. [43] contribuye a esta amplia área de computación en la nube al proponer un paradigma utilizando agentes que se pueden aplicar para mejorar el comercio entre los productores y los consumidores en cualquier industria, este trabajo es basado en una experiencia con una propuesto que comprende proporcionar una plataforma en un ambiente de nube en el que los agentes actúen en conjunto entre 
los clientes y los productores, analizando las solicitudes presentadas por los clientes y asignándolas al mejor productor adecuado.

En el campo del turismo [44] menciona la experiencia donde los servicios web semánticos necesitan una entidad de software superior capaz de tratar con ellos, por esta razón, se analiza la necesidad de integrar estas tecnologías y los beneficios potenciales de su combinación. El artículo [45] se desarrolló en el campo de la programación dónde se encuestó las investigaciones de Agentes Orientados a la Programación (AOP) desde tres aspectos: conceptos y modelos de programación, constructos de programación e idiomas, herramientas de programación y entornos, que son indispensables como paradigma de programación en ingeniería de software. Ya que la programación orientada a agentes (AOP) adopta conceptos y tecnologías de sistemas de agentes múltiples para desarrollar software.

Los trabajos de tipo metodología presentados pretenden relacionar un conjunto de procedimientos para que con la utilización se sistemas multi-agente se obtenga un procedimiento más sencillo y que sirva para gestionar tareas en empresas e industrias.

\subsection{En lo que respecta a contribuciones de tipo Herramienta}

Para [46] la tecnología del agente se aplica para tratar con el proceso de compra electrónica complejo, dinámico y distribuido, mientras que la tecnología del servicio web se aplica para brindar escalabilidad e interoperabilidad por esta razón en este documento, se ha propuesto un agente y una arquitectura basada en servicios web para el manejo de excepciones en e-procurement.

El documento [47] menciona que los sistemas multi-agente muestran una gran similitud con respecto a las cadenas de suministro, y brindan la flexibilidad para modelar las complejidades y dinámicas de las cadenas del mundo real sin excesivos supuestos simplificadores. Este trabajo es un experimento que tiene como objetivo desarrollar un holónico múltiples-agente para un aceite típico de la industria cadena de suministro. El trabajo [40] es una investigación que analiza el uso de agentes de software para ayudar a administrar la complejidad de los mercados de electricidad, particularmente los mercados minoristas.

El artículo [48] se desarrolla de una experiencia donde el desarrollo de un metalearner, aplicado al soporte de decisiones de las entidades de negociación de los mercados de electricidad, el metalearner propuesto ejecuta una red neuronal artificial dinámica para crear su propia salida, aprovechando varios algoritmos de aprendizaje 
implementados en ALBidS, un sistema de aprendizaje adaptativo que brinda apoyo a la toma de decisiones a los jugadores de los mercados de electricidad.

La investigación [49] propone un sistema flexible de programación para optimizar el cronograma del proyecto de construcción basado en la colaboración de entidades / socios en una cadena de suministro de construcción como caso de estudio. El trabajo [50] se desarrolló con base en una experiencia donde, los autores proponen un sistema basado en agentes llamado NM-MAS para la gestión de redes, con aplicación en el campo petrolero.

Los trabajos que se presentaron en el bloque anterior contribuyen con herramientas y sistemas que mediante el uso de sistemas multi-agente y aprovechando redes neuronales permita la toma de decisiones autónomas para procesos complejos.

\subsection{En lo que respecta a contribuciones de tipo descripción del área.}

[51] menciona que los sistemas multi - agente (MAS) proporcionan una arquitectura flexible para tratar con cambios basados en la organización dinámica y la colaboración de agentes autónomos. A pesar de los extensos estudios y resultados de investigación sobre MAS, el desarrollo de una metodología de diseño para apoyar la coordinación y las operaciones es fundamental para el éxito y la adopción de VE (virtual enterprises), los objetivos de esta investigación son proponer una metodología de diseño para facilitar la coordinación y el desarrollo de sistemas de gestión de flujo de trabajo sensibles al contexto y lograr una asignación de recursos efectiva para VE basada en la arquitectura MAS.

\subsection{En lo que respecta a contribuciones de tipo Modelo}

El artículo [53] es un experimento que para resolver el problema de distribución multitarea correspondiente, propone una solución usando diferentes enfoques aplicando los modelos de umbral de respuesta inspirados por la división del trabajo en insectos sociales, la aplicación del algoritmo de aprendizaje de refuerzo basado en el aprendizaje de la teoría de autómatas y algoritmos deterministas basados en la optimización de colonias de hormigas por lo que en este documento se centra en el problema general de la coordinación de múltiples robots, en particular, aborda el problema de la distribución de múltiples tareas heterogéneas de una manera robusta y eficiente. 
El documento [6] es una investigación donde se menciona que los sistemas MAS (Sistemas Multi Agente) proporcionan un paradigma para modelar estas características, las tareas de programación en MAS es un problema complejo debido a la arquitectura distribuida para programar tareas por agentes individuales y la dependencia de flujos de trabajo de diferentes agentes, este documento nos dice que se combina los sistema de arquitectura para descubrir dinámicamente servicios, flujo de trabajo y modelos de actividad para especificar las capacidades de los agentes, contratar protocolo de red para facilitar la negociación y coordinación de agentes y teorías de optimización.

El trabajo [3] es de tipo observación, en este contexto, el uso de agentes inteligentes para automatizar el proceso surge como una herramienta importante para facilitar esta actividad, este documento presenta pautas para la elaboración de un modelo de negociación basado en agentes para abordar el problema de seleccionar proveedores en el campo de la industria minorista. [52] es un caso de estudio donde se describen en detalle los problemas de agregación, forrajeo, agrupamiento y clasificación de objetos, navegación, formación de trayectorias, despliegue, manipulación colaborativa y asignación de tareas, por lo que nos explica que los principios de inteligencia de enjambre se han estudiado ampliamente y se han aplicado a una serie de tareas diferentes en las que se utiliza un grupo de robots autónomos para resolver un problema con un enfoque distribuido, es decir, sin coordinación central.

En los trabajos de tipo modelo se busca crear un tipo prototipo que permite ser referencia de procesos de la misma naturaleza creando un estándar mediante el uso de los sistemas multi-agente.

\section{Conclusiones}

En este trabajo, se caracterizó los sistemas multi-agentes a través de un estudio preliminar de mapeo en un conjunto de trabajos existentes. Se explicó una metodología de investigación adoptando las mejores prácticas que expone Petersen. Se presentó un esquema de clasificación para la categorización de los estudios, se mostró los resultados obtenidos y se hizo una reflexión inicial sobre los resultados. Este mapa sistemático muestra que la mayoría de las investigaciones han sido el desarrollo de procesos, metodologías, modelos y herramientas que permiten el trabajo con sistemas multi-agente.

El principal tipo de investigación son los casos de estudio, los cuales periten documentar la aplicación de sistemas multi-agente. Por último, mediante el análisis de los artículos se ha podido establecer que las áreas como la robótica, el control de 
producción y la aviación presentan importantes trabajos con sistemas multi-agente que ha permitido toma de decisiones adecuadas. Áreas de la computación como el software y el almacenamiento en la nube también han ganado mucho espacio aplicando sistemas multi-agente. Sin embargo, podríamos decir que el área de control de producción es una de la que más ha implementado la aplicación de los sistemas multi-agente. Vea tabla 7.

TABLE 7: Determinación de la población, la intervención y los resultados.

\begin{tabular}{l|c|}
\hline ÁREAS & NRO. \\
\hline E-Commerce & 4 \\
\hline Control de producción & 8 \\
\hline Industria Eléctrica & 5 \\
\hline Computación & 8 \\
\hline Aviación & 3 \\
\hline Robótica & 3 \\
\hline Otras & 11
\end{tabular}

\subsection{Amenazas de validez}

Las amenazas a la validez del estudio de mapeo se analizan de acuerdo a la siguiente taxonomía: validez de constructo, confiabilidad, validez interna y validez externa [16]. Este estudio representa una respuesta concreta a las preguntas de investigación por lo que se considera que tiene una validez constructiva. Los términos sistemas multi-agente, autonomía e inteligencia artificial, son suficientemente estables para ser utilizados como cadena de búsqueda.

Dado que el análisis sólo utiliza estadísticas descriptivas, las amenazas son mínimas. La clasificación de las investigaciones es una amenaza de validez interna ya que la selección de estudios está sujeta al sesgo del investigador, y el tiempo requerido para revisar y seleccionar artículos relevantes es un objetivo para la optimización [29, 54].

Finalmente, la validez externa es acerca de la generalización de este estudio [55]. Dado que no sacamos ninguna conclusión sobre los estudios de mapeo en general, sino sólo sobre este específico, las amenazas externas de validez no son aplicables.

\section{Discusión}

La evaluación, validación y evidencia empírica de los artículos indica que existe un alto volumen de investigación que se ha implementado en la práctica industrial. Los tipos de 
contribuciones reflejan que se ha realizado varios aportes en los sistemas multi-agente de tipo herramienta, proceso y modelos, aplicados a casos de industrias.

Con el fin de poder establecer el estado actual de la evidencia para la investigación de los sistemas multi-agente, enfatizamos la importancia de mejorar el uso de los métodos de investigación empíricos y aplicarlos en áreas aún no explotadas.

\section{References}

[1] R. Galán, Inteligencia Artificial, vol. 10. 2000.

[2] F. Zambonelli, N. R. Jennings, and M. Wooldridge, "Developing Multiagent Systems: The Gaia Methodology," ACM Trans. Softw. Eng. Methodol., vol. 12, no. 3, pp. 317-370, 2003.

[3] T. E. E. Goncalo and D. C. Morais, "Agent-Based Negotiation Protocol for Selecting Transportation Providers in a Retail Company," Proc. - 2015 IEEE Int. Conf. Syst. Man, Cybern. SMC 2015, pp. 263--267, 2016.

[4] S. Aknine, A Multi-Agent Model for Overlapping Negotiations, vol. 21, no. 6. 2012.

[5] H. Algarvio, F. Lopes, and J. Santana, "Multi-agent retail energy markets: Bilateral contracting and coalitions of end-use customers," Int. Conf. Eur. Energy Mark. EEM, vol. 2015--Augus, 2015.

[6] F. S. Hsieh and J. B. Lin, "A dynamic scheme for scheduling complex tasks in manufacturing systems based on collaboration of agents," Appl. Intell., vol. 41, no. 2, pp. 366--382, 2014.

[7] F. Lopes, H. Algarvio, and H. Coelho, "Agent-based simulation of retail electricity markets: Bilateral trading players," Proc. - Int. Work. Database Expert Syst. Appl. DEXA, pp. 189--193, 2013.

[8] V. J. Julián and V. J. Botti, "Estudio de métodos de desarrollo de sistemas multiagente," Rev. Iberoam. Intel. Artif., vol. Vol. 7, no. Num. 18, pp. 65--80, 2003.

[9] N. R. Jennings, "Building complex software systems: The case for an agent-based approach," Commun. ACM, vol. 44, no. Figure 1, pp. 35--41, 2001.

[10] V. A. Burégio, S. L. Meira, and E. S. De Almeida, "Characterizing Dynamic Software Product Lines -- A Preliminary Mapping Study," Sp/c, no. August 2015, pp. 53--60, 2010.

[11] S. Beecham, N. Baddoo, T. Hall, and H. Robinson, "Protocol for a Systematic Literature Review of Motivation in Software Engineering Systematic Review -- Cover Sheet," Computer (Long. Beach. Calif)., no. September, p. 87, 2006. 
[12] B. A. Kitchenham, D. Budgen, and O. Pearl Brereton, "Using mapping studies as the basis for further research - A participant-observer case study," Inf. Softw. Technol., vol. 53, no. 6, pp. 638--651, 2011.

[13] B. Kitchenham, "What's up with software metrics? - A preliminary mapping study," J. Syst. Softw., vol. 83, no. 1, pp. 37--51, 2010.

[14] B. Kitchenham and S. Charters, "Guidelines for performing Systematic Literature Reviews in Software Engineering," Engineering, vol. 2, p. 1051, 2007.

[15] A. Villar and S. Matalonga, "Definiciones y tendencia de deuda técnica: Un mapeo sistemático de la literatura," Memorias la XVI Conf. Iberoam. Ing. Softw. CIbSE 2013, pp. 33--46, 2013.

[16] K. Petersen, R. Feldt, S. Mujtaba, and M. Mattsson, "Systematic Mapping Studies in Software Engineering," 12Th Int. Conf. Eval. Assess. Softw. Eng., vol. 17, p. 10, 2008.

[17] X. I. Jics, "Calidad e Ingeniería del Software," Rev. Española Innov. Calid. e Ing. del Softw., pp. 3--27, 2010.

[18] G. Fernandez, "Metodología de la Investigación," p. 91, 2008.

[19] I. Ghani and I. Yasin, "Software Security Engineering In Extreme Programming Methodology: A Systematic Literature," Sci. Int., vol. 25, no. 2, pp. 215--221, 2013.

[20] E. Hossain, M. A. Babar, and H. Paik, "Using Scrum in Global Software Development: A Systematic Literature Review," 2009 Fourth IEEE Int. Conf. Glob. Softw. Eng., pp. 175--184, 2009.

[21] M. Bordons and M.. Ángeles Zulueta, "Evaluación de la actividad científica a través de indicadores bibliométricos," Rev. Española Cardiol., vol. 52, no. 10, pp. 790--800, 1999.

[22] C. Izurieta and J. M. Bieman, "How software designs decay: A pilot study of pattern evolution," Proc. - 1st Int. Symp. Empir. Softw. Eng. Meas. ESEM 2007, pp. 449--451, 2007.

[23] K. Petersen, R. Feldt, M. Shahid, and M. Mattsson, "Systematic Mapping Studies in Software Engineering," Ease'08, pp. 1--10, 2008.

[24] J. D. Velásquez, "Una Guía Corta para Escribir Revisiones Sistemáticas de Literatura Parte 3," Dyna, vol. 82, no. 189, pp. 9--12, 2015.

[25] A. Martínez, J. Ramírez, and A. Martínez-, “Estudio de Mapeo Sistemático sobre Priorización de Requerimientos de Software Basada en Valor Software Basada en Valor," no. July 2016, 2015.

[26] S. Mujtaba and K. Petersen, "Software product line variability: A systematic mapping study," Sch. Eng. ..., 2008. 
[27] R. Wieringa, N. Maiden, N. Mead, and C. Rolland, "Requirements engineering paper classification and evaluation criteria: A proposal and a discussion," Requir. Eng., vol. 11, no. 1, pp. 102--107, 2006.

[28] O. Dieste, A. Grimán, and N. Juristo, "Developing search strategies for detecting relevant experiments," Empir. Softw. Eng., vol. 14, no. 5, pp. 513--539, 2009.

[29] E. E. Hassler, D. P. Hale, and J. E. Hale, "A comparison of automated training-byexample selection algorithms for Evidence Based Software Engineering," Inf. Softw. Technol., vol. 98, no. February, pp. 59--73, 2018.

[30] Q. Baert, A. C. Caron, M. Morge, and J. C. Routier, "Fair multi-agent task allocation for large datasets analysis," Knowl. Inf. Syst., vol. 54, no. 3, pp. 591--615, 2018.

[31] B. Xie, S. Chen, J. Chen, and L. C. Shen, "A mutual-selecting market-based mechanism for dynamic coalition formation," Int. J. Adv. Robot. Syst., vol. 15, no. 1, pp. 1--10, 2018.

[32] B. L. Sadigh, S. Nikghadam, A. M. Ozbayoglu, H. O. Unver, E. Dogdu, and S. E. Kilic, "An ontology-based multi-agent virtual enterprise system (OMAVE): part 2: partner selection," Int. J. Comput. Integr. Manuf., vol. 30, no. 10, pp. 1072--1092, 2017.

[33] A. L. Blum and P. Langley, "Selection of relevant features and examples in machine learning," Artif. Intell., vol. 97, no. 1--2, pp. 245--271, 1997.

[34] N. Condori-Fernandez, M. Daneva, K. Sikkel, R. Wieringa, O. Dieste, and O. Pastor, "A Systematic Mapping Study on Empirical Evaluation of Software Requirements Specifications Techniques," 2009 3rd Int. Symp. Empir. Softw. Eng. Meas., no. 1, pp. 502--505, 2009.

[35] T. Pinto, T. M. Sousa, I. Praça, Z. Vale, and H. Morais, "Support Vector Machines for decision support in electricity markets' strategic bidding," Neurocomputing, vol. 172, pp. 438--445, 2016.

[36] M. Sichuan, "Automated Negotiation Model of Supply Chain Management Based on Multi-agent," Expert Syst., pp. 178--180, 2011.

[37] P. Ferreira, S. Doltsinis, A. Anagnostopoulos, F. Pascoa, and N. Lohse, “A performance evaluation of industrial agents," Ind. Electron. Soc. IECON 2013 - 39th Annu. Conf. IEEE, pp. 7404--7409, 2013.

[38] R. F??hring and S. Zelewski, "Towards decentralized electronic market places and agent-based freight exchanges for multimodal transports," IEEE Int. Conf. Autom. Sci. Eng., vol. 2015--Octob, pp. 249--254, 2015.

[39] S. L. Du, X. M. Sun, M. Cao, and W. Wang, "'Pursuing an evader through cooperative relaying in multi-agent surveillance networks," Automatica, vol. 83, pp. 155--161, 2017. 
[40] K. Ioannidis, G. C. Sirakoulis, and I. Andreadis, "Cellular ants: A method to create collision free trajectories for a cooperative robot team," Rob. Auton. Syst., vol. 59, no. 2, pp. 113--127, 2011.

[41] Z. Qin, Z. Lin, D. Yang, and P. Li, “'A task-based hierarchical control strategy for autonomous motion of an unmanned surface vehicle swarm," Appl. Ocean Res., vol. 65, pp. 251--261, 2017.

[42] H. Tang, D. Li, S. Wang, and Z. Dong, "CASOA: An architecture for agent-based manufacturing system in the context of Industry 4.0," IEEE Access, vol. 3536, no. c, 2017.

[43] S. Rajesh, K. Sundararajan, S. Padmavathi, S. Govindarajan, and S. Karthikeyan, "Genetic algorithmic approach for dynamic request processing in agent cloud platform," Souvenir 2015 IEEE Int. Adv. Comput. Conf. IACC 2015, pp. 1072--1076, 2015.

[44] E. Benaissa, B. Tahiri, and A. Benabdelhafid, "Applying multi-agent technique in cooperation: Case of tourism supply chain," 2013 Int. Conf. Adv. Logist. Transp. ICALT 2013, pp. 335--340, 2013.

[45] X. Mao and Q. Z. Wang, "A survey of agent-oriented programming: From program models to programming languages and environments," Proc. - 2015 IEEE/WIC/ACM Int. Jt. Conf. Web Intell. Intell. Agent Technol. WI-IAT 2015, vol. 2, pp. 159--165, 2016.

[46] S. X. Sun, J. Zhao, and H. Wang, "An agent based approach for exception handling in e-procurement management," Expert Syst. Appl., vol. 39, no. 1, pp. 1174--1182, 2012.

[47] F. J. M. Marcellino and J. S. Sichman, "A Holonic Multi-agent Model for Oil Industry Supply Chain Management," pp. 244--253, 2010.

[48] T. Pinto, T. M. Sousa, E. Barreira, I. Praca, and Z. Vale, "Metalearner based on dynamic neural network for strategic bidding in electricity markets," Proc. - Int. Work. Database Expert Syst. Appl. DEXA, vol. 2011, pp. 184--188, 2013.

[49] F. S. Hsieh, "Location-aware workflow scheduling in supply chains based on multiagent systems," TAAl 2015 - 2015 Conf. Technol. Appl. Artif. Intell., pp. 441--448, 2015.

[50] L. Ionita and I. Ionita, "NM-MAS: A multi-agent system for network management in oil industry," 2014 RoEduNet Conf. 13th Ed. Netw. Educ. Res. Jt. Event RENAM 8th Conf., pp. 1--6, 2014.

[51] F.-S. Hsieh and J.-B. Lin, "Context-aware workflow management for virtual enterprises based on coordination of agents," J. Intell. Manuf., vol. 25, no. 3, pp. 393--412, 2014. 
[52] L. Bayindir, "A review of swarm robotics tasks," Neurocomputing, vol. 172, pp. 292-321, 2016.

[53] J. de Lope, D. Maravall, and Y. Quiñonez, "Self-organizing techniques to improve the decentralized multi-task distribution in multi-robot systems," Neurocomputing, vol. 163, pp. 47--55, 2015.

[54] A. Sans and L. R. B. Atenea Alonso Serrano, Lorena García Sanz, Irene León Rodrigo, Elisa García Gordo, Belén Gil Álvaro, "Métodos de investigación de enfoque experimental," Metodol. la Investig. Educ., pp. 167--193, 2012.

[55] D. T. Campbell and J. C. Stanley, "Disenos experimentales y cuasiexperimentales en la investigación social," p. 79, 1966. 